\title{
A stochastic dynamic mass spectrometric diffusion method and its application to 3D structural analysis of the analytes
}

\author{
${ }^{1}$ Lehrstuhl für Analytische Chemie, Institut für Umweltforschung, Fakultät für Chemie und Chemische Biologie, Universität \\ Dortmund, Otto-Hahn-Straße 6, 44221 Dortmund, Nordrhein-Westfalen, Deutschland, Phone: +49 2317554089 , Fax: +49 231 \\ 75540 84, E-mail: B.Ivanova@infu.uni-dortmund.de. https://orcid.org/0000-0002-5788-4404. \\ ${ }^{2}$ Lehrstuhl für Analytische Chemie, Institut für Umweltforschung, Fakultät für Chemie und Chemische Biologie, Universität \\ Dortmund, Nordrhein-Westfalen, Deutschland
}

\begin{abstract}
:
There is a straightforward line in the recent development of the functional model connecting the experimental mass spectrometric variable intensity of a peak of an analyte ion with its thermodynamic, kinetic and diffusion parameters. It has been shown that the temporal behavior of the outcome intensity obeys a certain law: $\mathrm{D}_{\mathrm{SD}}=1.3193 \times 10^{-14} \times A \times \frac{\left(\bar{I}^{2}-(\bar{I})^{2}\right)}{(I-\bar{I})^{2}}$. This formula is universally applicable and empirically testable and verifiable. It connects the intensity with the so-called stochastic dynamic diffusion " $\mathrm{D}_{\mathrm{SD}}$ " parameter. Its application to small-scale research, so far, using soft-ionization electrospray, atmospheric pressure chemical ionization, matrix-assisted laser desorption/ionization or collision-induced dissociation methods has shown that the $\mathrm{D}_{\mathrm{SD}}$ parameter is linearly connected with the so-called quantum chemical diffusion parameter " $\mathrm{D}_{\mathrm{QC}}$," obtained within Arrhenius's theory. Therefore, the $\mathrm{D}_{\mathrm{SD}}$ parameter connects experimental measurable parameters of ions with their three-dimensional (3D) molecular and electronic structures. The corroborated empirical proof, so far, has convincingly argued that the mass spectrometry appears to be not only a robust instrumentation for highly accurate, precise and selective quantification but also is capable of providing the exact 3D molecular structure of the analytes, when it is used complementary to high accuracy methods of the computational quantum chemistry.
\end{abstract}

Keywords: 3D structural analysis, diffusion, mass spectrometry, quantum chemistry

DOI: 10.1515/revac-2019-0003

Received: April 25, 2019; Accepted: July 28, 2019

\section{Introduction}

The soft-ionization mass spectrometric (MS) electrospray ionization (ESI) and matrix assisted laser desorption/ionization (MALDI) methods have become indispensable analytical approaches over the recent decades. Their coupled instrumental schemes have become almost routinely implemented in analytical practice for peptidomics/proteomics, lipidomics, glycomics and glycoproteomics (Cooks et al., 1995; Ruhaak et al., 2018). We are unable to provide a comprehensive review of the literature because it is far beyond the scope of this paper. However, we will mention works that have applied MALDI and ESI methods in other interdisciplinary areas. MS protocols are compulsory in environmental research (Spiteller, 1981), agricultural sciences and food science and technology, including the area of methodological development of MS methods for food control (Wu \& Prior, 2005). MS methods have found application in the field of energetics (Ramirez-Pradilla et al., 2019), as well as in the field of environmental protection implementing new cleanup technologies (Kumar et al., 2018). An enormous amount of research effort has also been focused on the field of the chemistry of natural products. Argumentative examinations have emerged from other areas, for instance, those of forensic chemistry and public security. The motivation behind the broad use of MS methods in analytical practice is mainly due to superior instrumental characteristics.

We will, at this point, make an important remark that, in spite of a broad analytical implementation of MS protocols, the development of MS-based methods for multidimensional structural analysis is a challenging research task. From the perspective of methodological newcomers of MS, it is, in particular, very important 
to underline the novelty of our concept of quantitative treatment of the MS intensity based on a stochastic dynamic (SD) approach detailed in this review article and the crucial contribution of equation (1) to further methodological development and application of soft-ionization MS methods in analytical practice, in particular underlying the determination of the three-dimensional (3D) molecular conformation and electronic structure of the analytes. We highlight how further molecular level insights are captured by our new approach as well. In fact, we also underline what makes our new theoretical concept and model equation not only an attractive model but also a crucial concept of analytical quantitative and structural chemistry. In other words, what is unique in our innovative model connecting experimental measurable parameter intensity and physico-chemical properties of analytes, including their exact 3D molecular conformation and electronic structures?

The major aim of this review article is to provide the readers with an understanding of the formula of the measurable MS variable intensity based on stochastic dynamics (Ivanova \& Spiteller 2018a; 2018b; 2018c; 2019a; $2019 b ; 2019 c ; 2019 d)$.

$$
\mathrm{D}_{\mathrm{SD}}=1.3193 \times 10^{-14} \times A \times \frac{\left(\overline{I^{2}}-(\bar{I})^{2}\right)}{(I-\bar{I})^{2}}
$$

The crucial contributions are perhaps best understood in the light of other tools looking at equation (1) in comparison with known concepts of quantitative treatment of MS intensity. For this reason, the next section of the review begins with a description of our stochastic dynamic diffusion concept of the temporal behavior of the MS intensity of the analyte ions with respect to different spans of the measurement of time and some basic theories behind the derivation of the latter equation.

In the supporting information section we are confronted with the problem of the correlations between $\mathrm{D}_{\mathrm{SD}}$ and a kinetic " $k$ " parameter according to our other theory applicable to MS analyses, because of the fact that there is a functional relation between the diffusion and reaction kinetics that significantly extends the capability of the mass spectrometry as a robust analytical instrumentation for quantitative analysis. As this topic is outside the scope of the current review article, only some important references are detailed. The latter section illustrates quantitatively the correlation between $\mathrm{D}_{\mathrm{SD}}$ parameter and other known concepts of quantifying diffusion parameters by mass spectrometry. The relation between $\mathrm{D}_{\mathrm{SD}}$ and $\mathrm{D}_{\mathrm{CM}}$ parameters is described. The latter quantity is obtained experimentally by means of the so-called current monitoring method.

We do not enter into debates about known quantitative methods for treatment of MS diffusion parameters by other authors as they have been based on completely different methods. The highlights are on our new formula and its application to 3D structural analysis.

The major issues addressed in Section 3 fall under the problem of applied oriented aspects of the innovative concept of the quantitative description of the temporal behavior of the MS parameter intensity of an analyte ion and equation (1) under coupled experimental instrumental schemes of ESI (or APCI) with collision-induced dissociation (CID) mass spectrometry. There are examples (Section 4) of the application of this model equation to obtain experimental $D_{\mathrm{SD}}$ parameters (" $k$ ") of low molecular weight analytes, for instance, derivatives of piperazine, essential amino acids, oligopeptides and more, together with their metal-organic complexes with transition metal ions mainly with completed electronic configuration $\mathrm{d}^{10}$, for instance, $\mathrm{Ag}^{\mathrm{I}}$ and $\mathrm{Zn}^{\mathrm{II}}$ ions, in addition to complexes of $\mathrm{Cu}^{\mathrm{II}}$ ion. Chief among the discussion, therein, is the answer to a series of distinct research questions associated with mutual correlations between experimental MS parameters looking at $\mathrm{D}_{\mathrm{SD}}$ and $\mathrm{D}_{\mathrm{QC}}$ parameters. It is self-evident that a detailed account for such questions makes our theory for quantitative description of the temporal behavior of the intensity of the MS ions plausible. Because of the conjunction of experimental MS measurable parameter intensity with the absolute 3D molecular and electronic structures of the analyte, it is very peculiar from the perspective of the methods of the experimental analytical chemistry and other available analytical instrumentation for multidimensional structural analysis, for instance, the absolute method of single crystal X-ray diffraction, which provides direct information about the disposition of the atoms in 3D space based on chemometric methods for quantifying the experimental electron density of atoms.

The major point of our MS-based approach to 3D structural analysis with strongest evidential proof, so far, is that conceptually the MS parameter intensity provides direct information about the exact 3D molecular conformation and electronic structure of the ion via quantification of the exact energetics of the molecular structure. As far as the latter statement might be asserted, looking at basic theories of the chemical thermodynamics, what we have done in this review article is to detail quantitative correlations among $\mathrm{D}_{\mathrm{SD}} \Leftrightarrow \mathrm{D}_{\mathrm{OC}}$ parameters according to equation (1) in chemometric terms, encompassing a statistically representative set of MS ions of the analytes.

One of the key points made in the latter sections is that equation (1) is universally applicable not only to soft ionization methods using single operation modes, but also to multiple operation modes using coupled experimental schemes with CID-MS method, in addition to their application to a broad range of experimental conditions. In this way the content of the work focuses a significant amount of attention not only on the 
reliability of the equation across different soft-ionization methods, but also across a variety of coupling and hybrid schemes of analytical instrumentation, in particular, underlying the CID method. The latter instrumental design seemed to be advanced, amongst others, because it appears to be a significantly valuable approach to structural analysis of complex macromolecular objects. Therefore, the topics associated with methodological developments of methods for quantitative treatment of MS parameter intensity using experimental datasets of measurements in single operation mode together with tandem MS results are clearly among the most important problems for further research effort; besides that, they are straightforward. Obviously, our contribution to this field focusing on the discussed formula is applicable to a broad spectrum of instrumental methods, not only to the field of the analytical chemistry but also to the general field of analytical research.

To sum up, this review article attempts to illustrate persuasively that our model equation (1) of quantitative treatment of the experimental MS intensity determines exactly the 3D molecular and electronic structures of analyte ions when used complementary with high accuracy ab initio and density functional theory (DFT) methods of computational quantum chemistry within Arrhenius's theory. Results from analyses of amino acids, oligopeptides and their metal organics of ions of transition metals are discussed.

\section{Theory-formula connecting between the MS outcome intensity and the SD diffusion parameter}

The idea that the temporal behavior of the MS intensity must obey a central law has been a tenet of most methodological developments of formulas connecting measurable parameter intensity with parameters characterizing properties of analytes or those associated with the chemical reactions with participation of fragment ions or both. In line with such contributions, we discuss, herein, our innovative concept of the quantification of the latter experimental variable but using a completely new and purely SD diffusion approach, which has led to derivation of equation (1). We shall say that the crucial contribution of this formula to the domain of quantitative mass spectrometry lies in the fact that it appears applicable to a broad spectrum of different soft-ionization methods, for instance, ESI, MALDI, CID or APCI methods. Equation (1) connects experimental MS intensity (I) of $i$ th analyte ion within a given $j$ th span of the scan time of experimental measurement time with the $\mathrm{D}_{\mathrm{SD}}$ parameter. A summing up of the values of the absolute intensities with respect to the whole scan time of the measurement should provide the total intensity of analyte MS ion and the total SD diffusion parameter $\mathrm{D}^{\text {tot }}{ }_{\mathrm{SD}}$. Perhaps, still at this point it must be stressed that the results from the analyses discussed in the literature, so far, clearly provide experimental proof, not only of the validity of the latter formula but also for a quantitative correlation between $\mathrm{D}_{\mathrm{SD}}$ and the absolute total intensity "Itot" of the analytes and their MS ions. Statistically significant coefficients of correlation ranging from $r=0.9487$ to $0.9826_{9}$ have been obtained (Figure S1). Furthermore, this statistically significant correlation is valid to all the different MS ionization methods mentioned above.

In this subsection we determine briefly (including the scientific issues behind) the derivation of equation (1). Our theory is made up of a set of different assumptions starting out with a treatment of MS intensity with respect to different spans of the scan time as an SD variable. It is expressed quantitatively by adopting the Box-Müller's method (Gillespie, 1992; Satoh, 2011). According to equation (1), where " $x$ " = " $I$ ", and the BoxMüller's method, a stochastic variable " $x$ " (or $x=\mathrm{I}$ in our case) and its average value are connected with the so-called variance " $\sigma^{2}$ ". An assumption is that the distribution of the stochastic variable follows the MaxwellBoltzmann's distribution. We describe the absolute intensity of an analyte MS peak as a stochastic variable " $x$ " and $x=\mathrm{I}$, and the average value of the variable is given to be the average value $\bar{I}$ of the intensity but examining short spans of the measurement times. In other words, the concept is based on the description of the average value of the MS intensity, but per a concrete span of the scan time of the whole measurement time. The physical meaning of the parameter " $\sigma^{2}$ " is fluctuation. A parameter P1 represents a random number. However, the application of this method to quantify experimental MS intensity according to equation (1) has shown that $\ln \mathrm{P} 1$ is constant equal to $-15.053_{4}$. Let us recap that $\ln \mathrm{P} 1$ is constant per any span of scan time of the measurement. Logically, it is also constant per the span of the whole time of measurement. Looking at the latter equation and the functional relationship between MS intensity and time $(I-\bar{I})^{2}=f(t)$, it seems obvious that the experimental relations could be approximated to a nonlinear SineSqr function. The statistical parameter $A$ could be written as $A=-2 \sigma^{\prime 2} \cdot \ln P 1$ based on the original Box-Müller's method and SineSqr function. Accounting for the fact that, on the one hand, equation (1) has been found as a universally applicable model to a relatively representative set of different molecular objects and their fragment ions and, on the other hand, that $\ln P 1$ in the original Box-Müller's method is a constant, it is obvious that there must be a not only reliable but also a common physical meaning of the statistical parameter $A$, which is valid and applicable to all of the aforementioned MS experimental conditions. How do we develop this issue? We adopt the Ornstein-Uhlenbeck's approximation 
to variance parameter $\sigma^{2^{\prime}}=2 \beta^{2} \cdot \mathrm{D}_{\mathrm{SD}}$. We also apply the Einstein's approximation to the parameter $1 / \beta=3.10^{-8}$. On this basis we write equation (1). The data, so far, have shown that it is a verifiable and credible analytical approach to quantify experimental MS intensity regardless of the type of analyte ions and the ionization MS methods.

\section{Application of the SD approach to 3D structural analysis}

In the light of the topic of this review article, we will present a correlative analysis between $\mathrm{D}_{\mathrm{SD}}$ and $\mathrm{D}_{\mathrm{QC}}$ parameters according to equation (1) looking, in particular, at their application to 3D molecular structural analysis of MS ions (Figure 1 and Figure 2). As we do not have enough space in this work to go in-depth into many examples of the application of our SD concept of the MS quantification of the intensity and equation (1) to multidimensional structural analysis, we only highlight the very encouraging experimental support for the strategy unambiguously showing that $\mathrm{D}_{\mathrm{SD}}$ parameter corresponds linearly to the $\mathrm{D}_{\mathrm{OC}}$ parameter. The main motivation behind the strategy of this analysis is that the minimum value of the free Gibbs energy of any molecular system is a macroscopic quantity, which determines the most stable 3D molecular conformation, which in parallel is characterized by a unique electronic structure. The $\Delta \mathrm{G}$ value, therefore, depends on molecular structural and environmental factors. Given that, correlative analyses between free Gibbs energy values of different molecular systems enable to determine the exact multidimensional molecular structure (Chipot \& Pohorille, 2007) because the accuracy of the currently developed methods of the quantum chemistry is far below the uncertainty of many experimental analytical methods. In parallel, only soft-ionization MS methods among the currently available analytical instrumentation transfer charged species from condense phases to continuum without fragment reactions that should affect the 3D structure of the parent ions. Thus, the powerful capability of the MS methods consists of detailed molecular level information about covalent and noncovalent interactions in the condense phase, reflecting that singly and multiply charged ions of analyte molecules are produced in solution under ESI-MS conditions within a unimolecular reaction of protonation or deprotonation or both, thus excluding fragment processes. In view of these facts, it seems self-evident that only the MS methods are capable among the available instrumentation for 3D structural determination of providing exact multidimensional structural information in gas and condense phases by using our innovative SD method based on equation (1) complementary with high accuracy quantum chemical methods. The diffusion parameters according to the Arrhenius's approximation are obtained at a high accuracy by means of the latter methodology. According to the aforementioned view a linear correlation between the $\mathrm{D}_{\mathrm{SD}}$ and $\mathrm{D}_{\mathrm{QC}}$ parameters with a statistical significance is expected. The analyses, so far, show excellent statistical coefficients of correlation $r=0.9806_{8}\left(\mathrm{Cu}^{\mathrm{II}}-\mathrm{G} 5\right), 0.9901$ (G6), 0.95575 (H-Trp-Trp-OH), $0.9806_{8}-0.9956$ ( Ag $^{\mathrm{I}}$-containing metal organics), and 0.9833 (Cu ${ }^{\mathrm{II}}-\mathrm{Gly}_{\text {, }}$ ) respectively. Taking into consideration these results, we should highlight that the statistical analysis has been carried out by a far more detailed theoretical design and computations of a significantly larger set of molecular objects accounting for the effects of the conformational changes, subtle electronic effects, tautomerism, isomerism, polymorphism, intramolecular proton and charge transfer effects, and more. The discussed results are persuasive, credible and reliable. These analyses provide a backdrop to further systematic research and employment of this method for experimental determination of multidimensional molecular structure. The facts, so far, provide con arguments against the view that MS methods cannot be applied to 3D structural analysis (Veenstra, 1999; Song \& Spezia, 2018). However, what we have obtained as experimental evidence is that the method used in our theoretical concept and model formula allows for exact structural determination. It is controversial to claim that MS methods are restricted to application to 3D structural characterization (Veenstra, 1999; Song \& Spezia, 2018). In order to make our empirical pro arguments in a rigorous manner, let us describe an example of $3 \mathrm{D}$ structural analysis of a Cu${ }^{\mathrm{II}}$-complex with the amino acid glycine $\left(\left\{\left[\mathrm{Cu}^{\mathrm{II}}(\mathrm{Gly})_{2} \cdot 2 \mathrm{H}_{2} \mathrm{O}\right]\right\}\right)$ (Ivanova \& Spiteller, 2019a). The experimental scheme of the study is designed primarily to account for a mutual correlation between crystallographic and MS multidimensional conformations and geometry parameters. The same is true for the experimental proof of equation (1) over a statistically representative set of crystals of organic salts (Ivanova \& Spiteller, 2019e). Accounting for the fact that the crystallography appears to be an absolute approach to determine a multidimensional molecular structure in a solid state, our MS concept provides not only highly reliable $3 \mathrm{D}$ structural information but also sheds light on the chemical processes of the condense phase. Figure 2 depicts a crystallographic structural solution of complex $\left\{\left[\mathrm{Cu}^{\mathrm{II}}(\mathrm{Gly})_{2} \cdot 2 \mathrm{H}_{2} \mathrm{O}\right]\right\}$ together with $2 \mathrm{D}$ and $3 \mathrm{D}$ structures of MS ion at $m / z 212 / 214$. The latter is obtained due to cleavage of the axial ligands in the crystal structure of the complex under ESI-MS experimental conditions. Within a series of five possible 3D molecular and electronic structures of the ion, the following two geometries, $\mathrm{m}_{212 / 214 \_a}$ and $\mathrm{m}_{212 / 214_{c}}$, appear most probable from the perspective of the energetics. The latter structure corresponds to the minimum potential energy. Nevertheless, the difference in the energy of the two structures is $\Delta \mathrm{E}=|0.022021|$ a.u. or about $13 \mathrm{kcal} \cdot \mathrm{mol}^{-1}$. The former geometry corresponds to the molecular structure of the complex where there is only cleavage of the axial lig- 
ands, while the latter structure assumes not only a loss of the axial ligands but also a transition from a cis- to a trans-isomer. By contrast, with the $\Delta \mathrm{E}$ values shown above, the analysis of $\mathrm{D}_{\mathrm{SD}}$ parameters reveals a significant selectivity. The same is true for the $\mathrm{D}_{\mathrm{QC}}$ parameter, as far as the cis-isomers shows $\mathrm{D}_{\mathrm{SD}}=68.9722$, while the trans-isomers is characterized by $\mathrm{D}_{\mathrm{SD}}=9.4367 \mathrm{~cm}^{2} \cdot \mathrm{s}^{-1}$. Within a set of MS ions obtained due to fragment reactions of the coordination compound, the later geometry fits excellently to the chemometric linear equation connecting the $\mathrm{D}_{\mathrm{SD}}$ and $\mathrm{D}_{\mathrm{QC}}$ parameters. The comparative analysis among bond lengths and angles, including torsion and dihedral angles, shows that the difference in geometry parameters of the complex in the crystalline state and in solution obtained mass spectrometrically is $\Delta r=|0.023|-|0.028| \AA$ (consider $\Delta^{2}$ values in Figure 2). The difference in dihedral angles is $\Delta(\angle)=|6.2|-|9.0|^{\circ}$. Therefore, both cleavages of axial ligands from the inner coordination sphere and cis-/trans-isomerization are accompanied by the minimum of the change of the 3D molecular conformation of the ligand and corresponding electronic redistribution within the molecular skeleton.
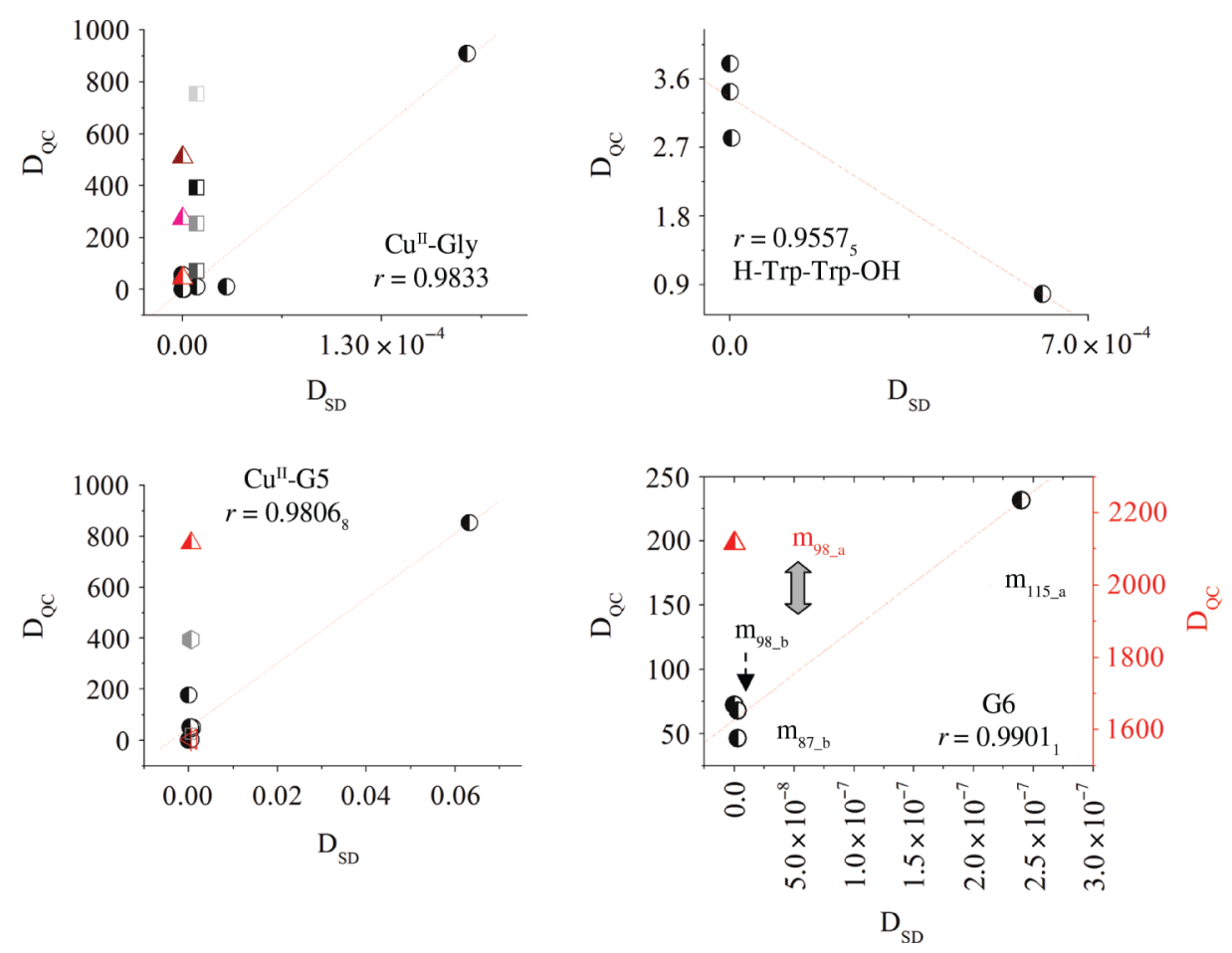

Figure 1: Quantitative linear correlations between the diffusion parameters $\mathrm{D}_{\mathrm{SD}}$ according to equation (1) of amino acids, oligopeptides and their metal organics and those obtained based on the Arrhenius's approximation, respectively; chemometrics; statistical coefficient of linear correlation " $r$ ". 

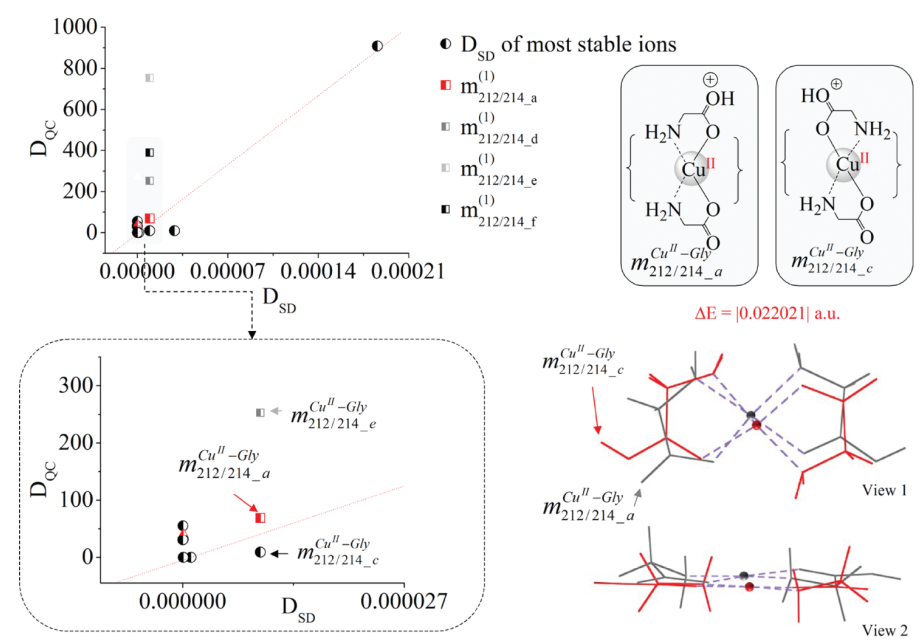

$m_{212 / 214 \_a}^{C u^{\prime \prime}-G l}$
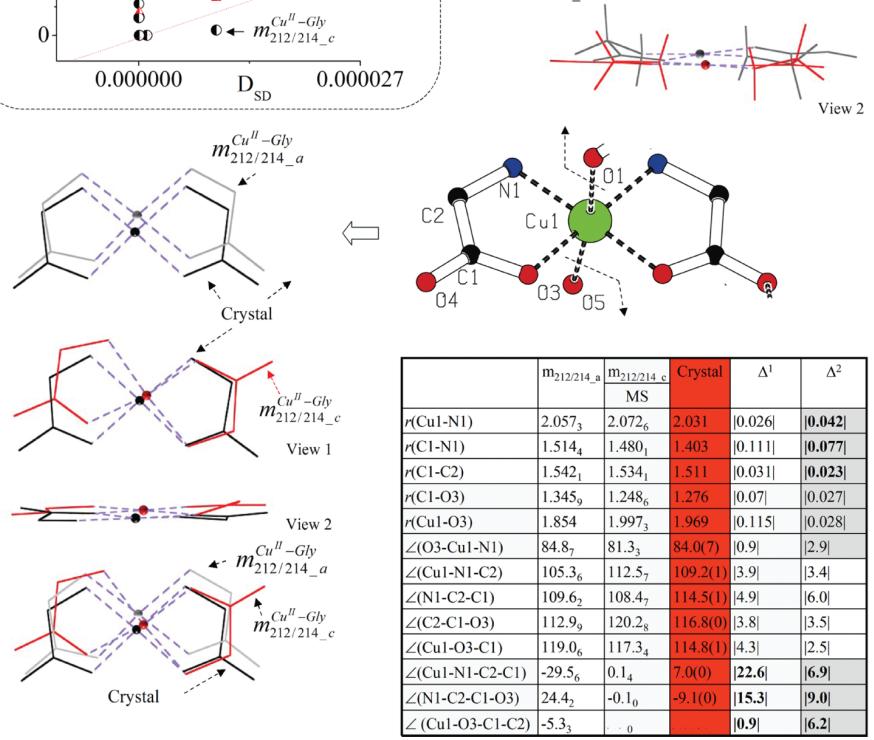

Figure 2: Relation between the $\mathrm{D}_{\mathrm{SD}}$ and $\mathrm{D}_{\mathrm{QC}}$ parameters $\left[\mathrm{cm}^{2} \cdot \mathrm{s}^{-1}\right]$ of MS ions of the complex $\left\{\left[\mathrm{Cu} \mathrm{u}^{\mathrm{II}}\left(\mathrm{Gly}_{2}\right)_{2} \cdot 2 \mathrm{H}_{2} \mathrm{O}\right]\right\}$ obtained based on equation (1) and the Arrenius's approximation to $\mathrm{D}_{\mathrm{QC}}$ parameter; crystallographic and mass spectrometrically determined 3D molecular structures of the complex together with the energetics $(\Delta \mathrm{E}$ [a.u.]) of most stable molecular conformations labeled as $\mathrm{m}_{212 / 214 \_a}$ and $\mathrm{m}_{212 / 214 \_c}$; selected geometry parameters, for instance, bond lengths [ $\mathrm{A}$ ], torsion and dihedral angles $\left({ }^{\circ}\right)$, respectively.

\section{Discussion}

The presentation of results from the preceding parts of this review article aims at providing empirical proof of not only the validity of equation (1) but also its great applicability to the exact multidimensional molecular and electronic structural determination of the analytes. Perhaps, the readers may accept only part of our pro arguments due to the fact that, as aforementioned, our concept has only been tested, so far, on small-scale research. In order to exclude rejection of our view based on possible sources of controversy between different interconnected theories behind formula (1), in this section we attempt to answer a question: Is there anything additional to be said about our method in spite of the fact that it appears to be an empirically testable approach?

So far, much attention has been concentrated on chemometric results from the correlation between $\mathrm{D}_{\mathrm{SD}}$ and $\mathrm{D}_{\mathrm{QC}}$ parameters, which has always obtained an excellent coefficient of correlations; based on this, we have claimed that the MS structural analysis is an exact method within our theory. Let us provide, first, some arguments and comments in defense of our statement.

The stochastic plausibility methods which we have adopted in writing equation (1) are exact tools. These theories account for true frequency, which means for the true value of the outcome parameter, which in our study appears as the MS intensity. It is quantified at concentration limits of detection at about $\sim 10^{-9}$ to $10^{-12} \mathrm{~mol} \cdot \mathrm{l}^{-1}$. It corresponds to a detectable number of particles at about $\sim 10^{16}$ to $10^{13}$. The fluctuations of the number of particles within the SD theories are obtained by means of the so-called $(\mathrm{N})^{1 / 2}$ law (Schuster, 2016). According to the latter law the fluctuation of the MS intensity at the concentration limit of detection should be within a range at about $(\mathrm{N})^{1 / 2} \sim 10^{8}$ to $10^{6}$. The accuracy of measurements does not account for fluctuations of $\sim 10^{6}$ particles. In other words, the SD theories describe, in general, exactly the experimentally observable outcome. 
Due to the limits of precision of the measurable outcome, the predictability of the instrumental response or the predictability of the variable is restricted. In other words, equation (1) is an exact formula. The same is true for the $a b$ initio and DFT methods used to obtain the $\mathrm{D}_{\mathrm{QC}}$ parameters. It is broadly acknowledged, based on a vast number of correlative analyses between theory and experiment, that these approaches provide highly reliable, accurate and precise information within the chemical accuracy. It is doubtless not controversial to claim that equation (1) is able to provide exact multidimensional MS-based structural determination of the molecules. Some comments on the highly competing capability of our method for 3D structural analysis compared with known robust analytical instrumentation for such purposes, for instance, single crystal X-ray diffraction, nuclear magnetic resonance (NMR) and circular dichroic (CD) spectroscopy should be carried out. In addition to, comments on the applicability of our approach to other powerful MS methods which have been broadly used for analytical practice, so far, but mainly to 2D structural analysis should be performed. Despite the fact that we have sought to determine multidimensional structures by a complementary application to single crystal X-ray diffraction and mass spectrometry, it is unquestionable that the structural analytical information is very distinguishable, as these are methods for condense phase analysis but in crystalline state and solution. In this context, the remarkable results from the analysis of the $\mathrm{Cu}^{\mathrm{II}}$ complex $\left\{\left[\mathrm{Cu}^{\mathrm{II}}(\mathrm{Gly})_{2} \cdot 2 \mathrm{H}_{2} \mathrm{O}\right]\right\}$ shown in the latter section clearly highlight that the complementary application of these two methods opens new doors in the comprehensive understanding of the chemistry of the condense phase, in particular, looking at the phase transition from solid state to solution. Thus, the available analytical instrumentation for structural analysis provides limited information, looking in particular at the capabilities of the NMR and CD spectroscopy (Li et al., 2017a, Li et al., 2017b). Despite the fact that multidimensional structural analysis by the latter methods involves high accuracy quantum chemical computations, the following major reason should be underlined at this place, because it significantly limits the applicability of these methods. The broad signals which they produce as analytical outcomes lead to a decrease in accuracy in determining the integral intensity of the signals. This directly affects the accuracy, in addition to a set of factors altering an accurate structural determination by NMR, which have been well determined currently as structure calculation problems of the method (Takashima, 2006). There has been enormous research effort on improvement of the quality of the multidimensional structural determination by NMR. However, the accuracy in determining dihedral angle values is at about $81 \%$ or there is a deviation of 19\%, furthermore, looking at high resolution NMR measurements (Armstrong, Kaas \& Rosengren, 2018; Jaremko et al., 2016). At this point, we are confronted, as well, with the problem of whether the MS concept should be more effective in studying the multidimensional molecular structure of analytes compared with an NMR analysis in solution. The assessment of the lastly shown accuracy of NMR measurements assumes that the error contribution from the experiment to the energy difference shown above between the cis-/trans-isomers of $\left\{\left[\mathrm{Cu}^{\mathrm{II}}(\mathrm{Gly})_{2} \cdot 2 \mathrm{H}_{2} \mathrm{O}\right]\right\}$ complex should be \pm 0.0044 a.u. A closer look at the energetics of all possible structures of the ion at $m / z 212 / 214$ (Ivanova \& Spiteller, 2019a) shows that the energy difference in isomers $\mathrm{m}_{212 / 214 \_\mathrm{d}}$ and $\mathrm{m}_{212 / 214 \_\mathrm{c}}$ is $\Delta \mathrm{E}=|0.014|$ a.u. It becomes more than clear that within a NMR-based structural determination there is inability to distinguish accurately among structures $\mathrm{m}_{212 / 214 \_d}, \mathrm{~m}_{212 / 214 \_a}$ and $\mathrm{m}_{212 / 214 \_c}$, respectively. On the contrary, within our theory as the latter work has shown, the $\mathrm{D}_{\mathrm{QC}}$ values vary largely with $\mathrm{D}_{\mathrm{QC}} \sim 9-253$ $\mathrm{cm}^{2} \cdot \mathrm{s}^{-1}$. We hope that this example is adequate evidence for the significant capability of the mass spectrometry for multidimensional structural analysis over NMR-based protocols. The difference in $\mathrm{D}_{\mathrm{SD}}$ values is even more underlined showing $\Delta \mathrm{D}_{\mathrm{SD}}=|485| \mathrm{cm}^{2} \cdot \mathrm{s}^{-1}$. We would also hold that the latter fact is additional evidence which can fully justify the statement that equation (1) provides exact 3D molecular and electronic structures. Not only the NMR method but also the single crystal X-ray diffraction and CD spectroscopy suffer from high sensitivity, when talking about their application to multidimensional structural analysis. Looking at biologically active biomacromolecules a set of conformations having closely disposed energies are very frequently found (Mistarz et al., 2016).

So far, this review has provided an innovative view of the crucial issue of the multidimensional structural application of soft-ionization MS methods; in what follows we explain why it is necessary to adequately distinguish between 2D and 3D structural determination when talking about an MS-based analysis. We should like to argue why the enormous research effort, so far, into the structural application of mass spectrometry has not achieve detailed 3D structural information. The direct answer seems to be that the current employment of mass spectrometry consists of assessment of mass-to-charge values of fragment ions yielding 2D, but not 3D molecular structural information. Because of this, as has been shown from our research, so far, the $\mathrm{D}_{\mathrm{SD}}$ parameter accounts for the multidimensional molecular conformation and electronic structure of the analyte ion. We may add that even with the employment of some of the most prominent MS methods, for instance, the ion mobility mass spectrometry, where the method operates within the spans of the measurement times between minutes and $\mu \mathrm{s}$ to $\mathrm{ms}$, the MS structural information is mainly limited to 2D analysis as has been shown from the accumulated research more recently (Fuller et al., 2018; Beveridge et al., 2019; Chen et al., 2019; Woodall et al., 2019). The methodological efforts consisting of the computation of collision cross section (CCS) yields, in fact, to energetics of analyte ions, presumably corresponding to a 3D molecular conformation, which is con- 
nected with the drift time and the velocity of the MS ions via the Mason-Schamp equation. However, as can be seen from the example presented in the preceding section, the energy difference in the molecular conformations can be significantly lower, which prevents an accurate determination of the exact multidimensional molecular structure, because the aforementioned CCS does not correspond to the real CCS. The molecular CCS reflects average values of the geometry orientation of the analyte within the whole time of measurement (May, Morris \& McLean, 2017; El-Baba et al., 2018; Conant et al., 2019). However, as our research, so far, has shown, the molecular conformation and the electronic interactions can be perturbed due to solute-continuum interactions under the MS experiment. The employment of isotope H/D exchange techniques allows for determining structural isomers and even enantiomers (Rob et al., 2012; Mistarz et al. 2014; 2016; Li et al. 2017a; 2017b), but with a significantly reduced accuracy due to the well-known phenomena associated with the kinetics of the H/D-exchange (Iacob \& Engen, 2012). Given that, a detailed analysis of the short spans of measurement times enables us to account accurately and precisely for the 3D structure. It is self-evident, in this context, that a quantification of $\mathrm{D}_{\mathrm{SD}}$ parameters per different short spans of scan time appears to be another significant advantage of our method, among others. Of course, the latter statement does not reject the capability of the above mentioned methodological MS contributions mainly to the 2D structural analysis. On the contrary, the fact that the ion mobility MS method provides information about the velocity of the analyte MS ion makes equation (1) a method directly applicable to this MS approach, as far as there is a well-known relation between velocity and the diffusion of charged species within the SD theories. What does the latter statement mean? It means that we assume with a high probability that our equation (1) is directly applicable to the ion mobility MS measurable outcome of the intensities of the analyte ions. The same is true for the fast H/D exchange MS operating within a sub-millisecond span of time after ESI-MS method. Its basic assumption involves monitoring of backbone amide H/D dynamics (Rob et al., 2012; Mistarz et al. 2014; 2016; Li et al. 2017a; 2017b). In a analogy with our methodology, the latter approach implements molecular dynamic simulations, in order to distinguish between most preferred conformations of the analyte with respect to the minimum of the potential energy. However, through the whole text of the paper we have underlined that only an assessment of the energy of the molecular systems is unable to distinguish precisely between different conformations (Conant et al., 2019). As mentioned before, the employment of $\mathrm{D}_{\mathrm{SD}}$ parameter, due to its great sensitivity, enables us to determine exactly the 3D molecular and electronic molecular structures based on soft-ionization MS experiment.

\section{Conclusion}

The problem of employing methods of mass spectrometry for the exact multidimensional determination of the molecular and electronic structures of analytes is, undeniably, one of the most explicit and debatable topics in tion for structural analysis. Although the methods of mass spectrometry have been proven over decades as being indispensable and irreplaceable analytical instrumentation for qualitative and quantitative analyses which already have found a place in different interdisciplinary research fields, there remains the question whether they can be employed for exact 3D structural analysis. Furthermore, the latter statement can be regarded as a common view currently. However, the main concept of quantitative analysis of the MS measurable parameter "intensity" that we present in this review article is that the temporal behavior of the later variable obeys the certain law [Equation (1)]. It provides direct link between the experimental outcome "intensity" and the diffusion parameter " $\mathrm{D}_{\mathrm{SD}}$ " of the ionic species determining their properties. The results in our more recent works have shown that the $\mathrm{D}_{\mathrm{SD}}$ parameter correlates linearly with the diffusion parameter determined within the framework of Arrhenius's theory $\left(\mathrm{D}_{\mathrm{QC}}\right)$. Importantly, the $\mathrm{D}_{\mathrm{SD}}$ parameter provides the bridge between experimental measurable MS outcome of analyte ion and its 3D molecular and electronic structures, so far; the $\mathrm{D}_{\mathrm{QC}}$ reflects a unique energy value which corresponds to the unique 3D molecular conformation and electronic structure of the molecular system. Importantly, our model equation equation (1) appears a universally applicable and empirically testable model. It is a verifiable and credible analytical approach to determine quantitatively experimental MS intensity of any type of analytes by different soft-ionization methods. Rather, we discuss the experimental facts about correlations between $\mathrm{D}_{\mathrm{SD}}$ and $\mathrm{D}_{\mathrm{QC}}$ parameters as underprintings that should allow the reader to look at the soft-ionization methods not only as a robust analytical instrumentation for quantification, but also, in fact, as a powerful analytical tool for experimental 3D structural determination of the molecules. Furthermore, it is a method applicable to both gas and condense phases. The instrumental characteristics of the methods of mass spectrometry that we discuss in this work and the derivation of equation (1) extends the analytical capability of the MS methods far beyond their current analytical employment. As we have shown, our contributions, so far, deal with studies that arrived out of the routine field of quantitative MS-based analysis. On the contrary, our innovative model equation and the burdens of empirical proof of their validity and appli- 
cability to analysis of molecular systems have raised new prospective areas of analytical implementation of the mass spectrometry into the exact multidimensional structural analysis. These methodological developments therefore have become increasingly important not only for the sub-field of the "analytical chemistry" dealing with "quantitative analysis" but also for the sub-field of the "structural analytical chemistry".

\section{Supporting information}

Chemometric analyses of functional relations between $\mathrm{D}_{\mathrm{SD}}$ and other kinetic and thermodynamic parameters; method performances (Figures S1-S6).

\section{Acknowledgments}

We are grateful to Deutscher Akademischer Austausch Dienst, the Alexander von Humboldt Stiftung, the Deutsche Forschungsgemeinschaft (DFG), the central instrumental laboratories for structural analysis at Dortmund University (Nordrhein-Westfalen, Germany) and analytical and computational laboratory clusters at the Institute of Environmental Research at the same University (Project: 224755478).

\section{Funding}

Michael Spiteller has received a research grant (DFG, Funder Id: http://dx.doi.org/10.13039/501100001659 255/22-1); Bojidarka Ivanova has received a research grant (DFG 255/22-1).

Conflict of interest: The review article was carefully carried out. Despite this, the authors and publisher do not claim the information therein to be free of errors.

\section{References}

Armstrong, D.; Kaas, Q.; Rosengren, K. Prediction of disulfide dihedral angles using chemical shifts. Chem. Sci. 2018, 9, $6548-6556$.

Beveridge, R.; Migas, L.; Kriwacki, R.; Barran, P. Ion mobility mass spectrometry measures the conformational landscape of p27 and its domains and how this is modulated upon interaction with Cdk2/cyclin A. Angew. Chem. Int. Ed. 2019, 58, 3114-3118.

Chen, X.; Raab, S.; Poe, T.; Clemmer, D.; Larriba-Andaluz, C. Determination of gas-phase ion structures of locally polar homopolymers through high-resolution ion mobility spectrometry-mass spectrometry. J. Am. Soc. Mass Spectrom. 2019, 30, 905-918.

Chipot, C.; Pohorille, A. (Eds.) Free Energy Calculations; Springer Verlag: Berlin Heidelberg, 2007.

Cooks, R.; Cleven, C.; Horn, L.; Nappi, M.; Weil, C.; Soni, M.; Julian, R. Non-destructive detection of ions in a quadrupole ion trap using a d.c. pulse to force coherent ion motion: a simulation study. Int. J. Mass Spectrom. 1995, 146/147, 147-163.

Conant, C.; Fuller, D.; El-Baba, T.; Zhang, Z.; Russell, D.; Clemmer, D. Substance P in solution: trans-to-cis configurational changes of penultimate prolines initiate non-enzymatic peptide bond cleavages. J. Am. Soc. Mass Spectrom. 2019, 30, 919-931.

El-Baba, T.; Fuller, D.; Hales, D.; Russell, D.; Clemmer, D. Solvent mediation of peptide conformations: polyproline structures in water, methanol, ethanol, and 1-propanol as determined by ion mobility spectrometry-mass spectrometry. J. Am. Soc. Mass Spectrom. 2018, 30, 77-84.

Fuller, D.; Conant, C.; El-Baba, T.; Brown, C.; Woodall, D.; Russell, D.; Clemmer, D. Conformationally regulated peptide bond cleavage in bradykinin. J. Am. Chem. Soc. 2018, 140, 9357-9360.

Gillespie, D. Markov Processes; Academic Press: NY, 1992.

lacob, R.; Engen, J. Hydrogen exchange mass spectrometry: are we out of the quicksand? J. Am. Soc. Mass Spectrom. 2012, 23, $1003-1010$.

Ivanova, B.; Spiteller, M. Experimental and theoretical mass spectrometric quantification of diffusion parameters and 3D structural determination of ions of L-tryptophyl-L-tryptophan in electrospray ionization conditions in positive operation mode. J. Mol. Struct. 2018a, 1173, 848-864.

Ivanova, B.; Spiteller, M. An Experimental and Theoretical Mass Spectrometric Quantification of Non-covalent Interactions in High Order Homogeneous Self-associates of Nucleobases and Nucleosides; NOVA Science Publishers: New York, $2018 \mathrm{~b}$.

Ivanova, B.; Spiteller, M. Quantification by Matrix-Assisted Laser Desorption lonization Mass Spectrometry Using an Approach Based on Stochastic Dynamics. Experimental and Theoretical Correspondences; GRIN Verlag: Muenchen, 2018c.

Ivanova, B.; Spiteller, M. 3D structural analysis of copper(II) complex of glycine-experimental mass spectrometric and theoretical quantum chemical approach. J. Mol. Struct. 2019a, 1179, 192-204.

Ivanova, B.; Spiteller, M. Experimental mass spectrometric and theoretical treatment of the effect of protonation on the 3D molecular and electronic structures of low molecular weight organics and metal-organics of silver(I) ion. In: Protonation: Properties, Applications and Effects; Cermogen, A., Ed.; Nova Science Publishers: New York, 2019b. 
Ivanova, B.; Spiteller, M. Stochastic dynamic electrospray ionization mass spectrometric diffusion parameters and 3D structural analysis of coordination species of copper(II) ion with glycylhomopentapeptide and its dimeric associates. J. Mol. Liq. 2019c, 282, 70-87.

Ivanova, B.; Spiteller, M. Electrospray lonization and Collision Induced Dissociation Mass Spectrometric Quantitative Conjunctions with the Experimental Intensity of the Analyte lons of Metal-organics - Stochastic Dynamics; GRIN Verlag: Muenchen, 2019d.

Ivanova, B.; Spiteller, M. Mass spectrometric experimental and theoretical quantification of reaction kinetics, thermodynamics and diffusion of piperazine heterocyclics in solution. In Advances in Chemistry Research; Taylor, J., Ed.; NOVA Science Publishers Inc.: NY, 2019e; Vol. 48; pp. 1-82, ISBN: 978-1-53614-724-7.

Jaremko, M.; Jaremko, L.; Villinger, S.; Schmidt, C.; Griesinger, C.; Becker, S.; Zweckstetter, M. High-resolution NMR determination of the dynamic structure of membrane proteins. Angew. Chem. Int. Ed. 2016, 55, 10518-10521.

Kumar, U.; Caikwad, V.; Mayyas, M.; Bucknall, M.; Sahajwalla, V. Application of high-resolution NMR and CC-MS to study hydrocarbon oils derived from noncatalytic thermal transformation of e-waste plastics. ACS Omega 2018, 3, 9282-9289.

Li, G.; Kusari, S.; Golz, C.; Laatsch, H.; Strohmann, C.; Spiteller, M. Epigenetic modulation of endophytic Eupenicillium sp. LG41 by a histone deacetylase inhibitor for production of decalin-containing compounds. J. Nat. Prod. 2017a, 80, 983-988.

Li, J.; Lyu, W.; Rossetti, G.; Konijnenberg, A.; Natalello, A.; Ippoliti, E.; Orozco, M.; Sobott, F.; Crandori, R.; Carloni, P. Proton dynamics in protein mass spectrometry. J. Phys. Chem. Lett. 2017b, 8, 1105-1112.

May, J.; Morris, C.; McLean, J. Ion mobility collision cross section compendium. Anal. Chem. 2017, 89, 1032-1044.

Mistarz, U.; Brown, J.; Haselmann, K.; Rand, K. Simple setup for gas-phase H/D exchange mass spectrometry coupled to electron transfer dissociation and ion mobility for analysis of polypeptide structure on a liquid chromatographic time scale. Anal. Chem. 2014, 86, 1186811876.

Mistarz, U.; Brown, J.; Haselmann, K.; Rand, K. Probing the binding interfaces of protein complexes using gas-phase H/D exchange mass spectrometry. Structure 2016, 24, 310-318.

Ramirez-Pradilla, J.; Blanco-Tirado, C.; Hubert-Roux, M.; Giusti, P.; Afonso, C.; Combariza, M. Comprehensive petroporphyrin identification in crude oils using highly selective electron transfer reactions in MALDI-FTICR MS. Energy Fuels 2019, 33, 3899-3907.

Rob, T.; Liuni, P.; Gill, P.; Zhu, S.; Balachandran, N.; Berti, P.; Wilson, D. Measuring dynamics in weakly structured regions of proteins using microfluidics-enabled subsecond H/D exchange mass spectrometry. Anal. Chem. 2012, 84, 3771-3779.

Ruhaak, L.; Xu, G.; Li, Q.; Coonatilleke, E.; Lebrilla, C. Mass spectrometry approaches to glycomic and glycoproteomic analyses. Chem Rev. 2018, 118, 7886-7930.

Satoh, A. Introduction to Practice of Molecular Simulation; Elsevier: Amsterdam, 2011.

Schuster, P. Stochasticity in Processes, Fundamentals and Applications to Chemistry and Biology; Springer Verlag: Berlin Heidelberg, 2016.

Song, K.; Spezia, R. Theoretical Mass Spectrometry; De Gruyter: Berlin Boston, 2018.

Spiteller, M. Abbauprodukte von Huminsäuren und ihre Identifizierung durch Kapillargaschromato-graphie-Massenspektrometrie, Mitteilungen der Dtsch. Bodenkundl. Cesellsch. 1981, 32, 541-550.

Takashima, H. High-resolution protein structure determination by NMR. Ann. Rep. NMR Spectrosc. 2006, 59, 235-273.

Veenstra, T. Electrospray ionization mass spectrometry in the study of biomolecular non-covalent interactions. Biophys. Chem. 1999, 79, 6379.

Woodall, D.; El-Baba, T.; Fuller, D.; Liu, W.; Brown, C.; Laganowsky, A.; Russell, D.; Clemmer, D. Variable-temperature ESI-IMS-MS analysis of myohemerythrin reveals ligand losses, unfolding, and a non-native disulfide bond. Anal. Chem. 2019, 91, 6808-6814.

Wu, X.; Prior, R. Systematic identification and characterization of anthocyanins by HPLC-ESI-MS/MS in common foods in the United States: fruits and berries. J. Agric. Food Chem. 2005, 53, 2589-2599.

Supplementary Material: The online version of this article offers supplementary material (DOI: https://doi.org/10.1515/revac-2019-0003). 